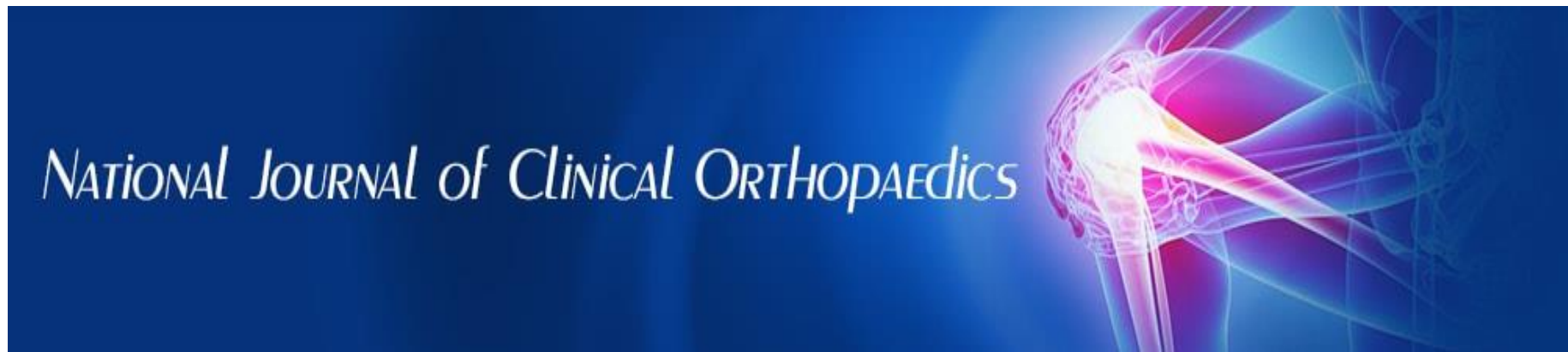

ISSN (P): 2521-3466

ISSN (E): 2521-3474

(C) Clinical Orthopaedics

www.orthoresearchjournal.com

2021; 5(3): 96-105

Received: 19-05-2021

Accepted: 21-06-2021

Guruprasath Arunagiri

Senior Assistant Professor,

Department of Orthopaedic

Surgery, Govt. Stanley Medical

College, Chennai, Tamil Nadu,

India

Sathish Kumar Thangamani

Assistant Professor, Department

of Orthopaedic Surgery, Govt.

Stanley Medical College,

Chennai, Tamil Nadu, India

Lalith Jagat

J Resident, Department of

Orthopaedic Surgery, Govt.

Stanley Medical College,

Chennai, Tamil Nadu, India

Tholgapiyan Thirueni

Prof. \& HOD, Department of

Orthopaedic Surgery, Govt.

Stanley Medical College,

Chennai, Tamil Nadu, India
Corresponding Author: Guruprasath Arunagiri Senior Assistant Professor,

Department of Orthopaedic

Surgery, Govt. Stanley Medical

College, Chennai, Tamil Nadu,

India

\section{Functional outcome of arthroscopic posterior cruciate ligament reconstruction using quadrupled semitendinosus gracilis graft: A prospective study}

\author{
Guruprasath Arunagiri, Sathish Kumar Thangamani, Lalith Jagat and \\ Tholgapiyan Thirueni
}

DOI: https://doi.org/10.33545/orthor.2021.v5.i3b.298

\section{Abstract}

Background: Posterior cruciate ligament (PCL) is strongest of all ligaments around knee \& its reconstruction remains a technically demanding surgery. Outcome of the surgery is multifactorial including graft material Selection, method of fixation etc. Our prospective study evaluates results of PCL reconstruction using quadripuled semitendinoses gracilis autograft, with 3 years follow up.

Patients and Methods: 40 patients were included in the study which extended for 36 months. This was a prospective study and all cases which had PCL rupture during this period were considered for this study. Patients with isolated chronic PCL rupture as well as multi ligament injuries including PCL rupture were included in the study.

Results: Physiotherapy was started on the second postoperative day and $40 \%$ had excellent outcome with Lysholm knee score whereas $60 \%$ had good outcome. Majority of the patients (95\%)were men which implies that this kind of injuries common with RTA's and worksite injuries.

Conclusion: Reconstruction of PCL using quadripuled semitendinoses gracilis autograft improves the clinical outcome drastically.

Keywords: quadrupled semitendinosus graciles graft, posterior cruciate ligament, reconstruction

\section{Introduction}

Knee injuries are more common nowadays due to increase in involvement in athletic activities, sports activities, Motor vehicle accidents. Posterior cruciate ligament is the strongest ligament and it is twice as strong as anterior cruciate ligament. PCL tears are quite uncommon when compared to ACL tears \& among the most serious ligament injuries.

A study shows that patients with knee injury had at least five times increased risk of developing early osteoarthritis of knee ${ }^{1}$ with PCL injuries

Nowadays PCL reconstruction has become choice for treatment for posterior laxity of knee. However, it is still not clear whether PCL reconstruction will improve subjective symptoms or prevent osteoarthritis compared with non operative treatment ${ }^{[2]}$.

Natural history of PCL disruption is still debated, some investigator describes untreated patients with minimal symptom presents with significant osteoarthritic changes later with PCL insufficiency in exceptionally high numbers when repair or reconstruction was delayed beyond 4 years ${ }^{[3]}$.

Most frequent injury mechanism causing PCL tear is direct blow on anterior tibia with flexed knee eg: Dashboard injury and soccer injury. Previously before the advent of arthroscopy surgical techniques ligament reconstruction surgeries were performed by open methods which itself was a complex procedure, prolonged time of procedure, risk of infection, high surgical site morbidity and difficult rehabilitation for patients.

After the advent of arthroscopy surgeries it is the preferred choice of treatment for ligaments injuries and intraarticular pathology of knee joint involving menisci, cartilage, synovium etc. 


\section{Aims \& Objectives}

To evaluate the functional outcome of arthroscopic reconstruction of posterior cruciate ligament injury using quadrupled semitendinosus gracilesauto graft.

\section{Methodology}

This is a prospective study conducted in Government Stanley Hospital Chennai in duration between June 2017 to May 2020.

In this study 40 patients were operated with PCL reconstruction. In this study $95 \%$ (38) patients were male and 5\% (2) were female. Right side injury (75\%) is predominant in this study than left side injury (25\%). The patients were followed up post operatively for average duration of 15.22 months and functional outcome was evaluated using IKDC and LYSHOLM score and PCL STRESS VIEW $x$ ray serially.

All the patients with history of trauma to knee joint, complaining of pain, instability presented in emergency department and out patients department of Orthopedics and Traumatology in Government Stanley Hospital Chennai were properly examined and evaluated and admitted as per patients general conditions.

In emergency department patients are thoroughly examined with detailed history and routine investigation has been done. Patients general conditions were stabilized and other vital injury were ruled out. Initially long knee immobilizer brace applied and measures taken to control pain and swelling. MRI has been taken for each patient to assess ligament injuries. Standard tests like Posterior drawer test, Quadriceps active test, Godfrey test and Reverse Lachman test were performed to clinically asses PCL injury

\section{Inclusion criteria}

All the patients with posterior cruciate ligament insufficiency from any age group and sex.

\section{Exclusion criteria}

- Compound grade injury to knee

- Local infection

- Obese patients having BMI > 40

- Patient medically unfit for surgery

- Patient not willing for operative treatment.

\section{Pre-operative investigation}

CBC, RBS, RFT, Chest $x$ ray, knee $x$ ray, PCL stress knee $x$ ray, MRI of knee, Doppler of lower limb in case of dislocated knee, HIV, HbsAg, HCV markers, Echocardiogram for patients older than 40 year, all necessary clearance and opinion required for anesthesia fitness for surgery.

\section{Pre-operative rehabilitation}

All the patients were taken adequate rest for minimum 21 days for settlement of edema and inflammation.

Pre operative movement of both knee and strength was assessed. Quadriceps strengthening exercise, knee mobilization exercise given.

\section{Choice of graft}

In our study we preferred to use quadripuled semitendinoses gracilis autograft since the graft can be harvested using very small skin incision of about $3 \mathrm{~cm}$ without compromising extensor apparatus and have very high elastic modulus

\section{Anesthesia}

Spinal anesthesia with Pneumatic tourniquet was preferred.

\section{Surgical prophylaxis}

$1 \mathrm{gm}$ Ceftriaxoneis given pre operatively before inflation of tourniquet.

\section{Examination under anesthesia}

Underanesthesia, posterior drawer test, anterior drawer test, Lachman test, pivot shift test, varus and valgus stress test are performed.

\section{Surgical technique}

Diagnostic arthroscopy is done prior to procedure.

\section{Graft harvesting and graft preparation}

Harvesting of Semitendinous graft ${ }^{\text {[52] }}$

1. Palpation: over the anteromedial aspect of proximal tibia 1 to $3 \mathrm{~cm}$ below and $1 \mathrm{~cm}$ medial to tibial tuberosity. pes anserinus is palpable in thin patents.

2. Skin incision: Oblique skin incision given about 5 to $10 \mathrm{~mm}$ below from tibial tuberosity on anteromedial side and extending 2.5 to $3 \mathrm{~cm}$ distally.

3. Exposure of pes anserinus: Subcutaneous tissue is dissected and pes anserinus is exposed, superficial layer is aponeurosis tendon of Sartorius muscle. Deep to Sartorius, gracilis and semitentinosus is palpated and identified

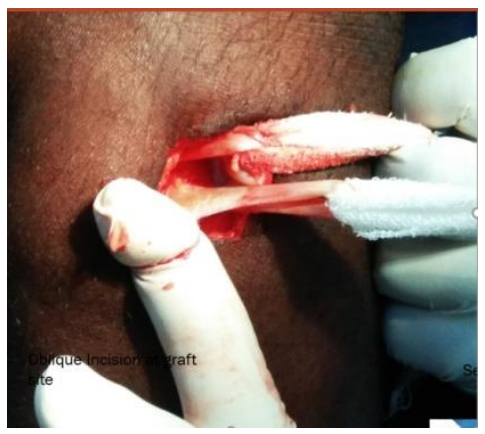

Image showing hooked ST and Gracilis tendon

4. Harvesting of tendon: It is a quiet difficult and time taking step as we need to identify and harvest ST tendon. Below the aponeurotic attachment of Sartorius, ST and gracilise will be located. Gracilis will be proximally and ST will be distally situated. Gracilis will be smaller thin cord like structure and ST will be flat, less homogenous structure.

5. Harvesting technique: Sartorius tendon is incised proximal to gracilis tendon, exposing the bursa below the pes anserinus, tendon is felt with finger. Using right angled artery forceps both tendons are hooked out, tendon ends are tiedwith knots using vicryl suture. Knee is flexed and proximal dissection done using finger till musculotendinous junction adhesion is released. Distal end of tendon is cut with scissor and tendon stripper is used with firm, continuous, gentle pressure and traction is applied from holding the thread knot. Any Adhesionfelt is released, stripper is advanced and tendon is harvested. Harvested graft is carefully placed on board and the tendon is processed by removing muscle fiber from tendon. The graft after quadrupling,

Whipstitches are applied at both ends of tendon. 3 to $4 \mathrm{~cm}$ of both ends of tendon stitched together. The composite graft is passed from graft sizer. Diameter of tunnel to be created should be smaller size sleeve through which quadruple graft should pass with minimum friction. 


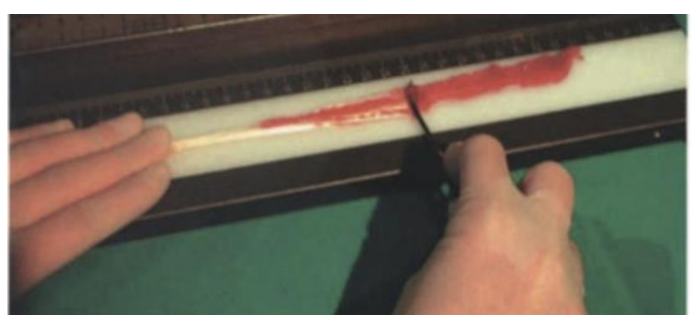

Image showing removal of muscle fiber from harvested graft

\section{Preperation of Graft}

Harvested graft is prepared and quadrupled, whip stitches applied. Tensioning is done using tendon board at $10 \mathrm{lbs}$ tension for 15 minutes.

The tendon can be folded in loop. It can be folded over to create four stranded graft. Tendon must be cleared from muscle fiber.

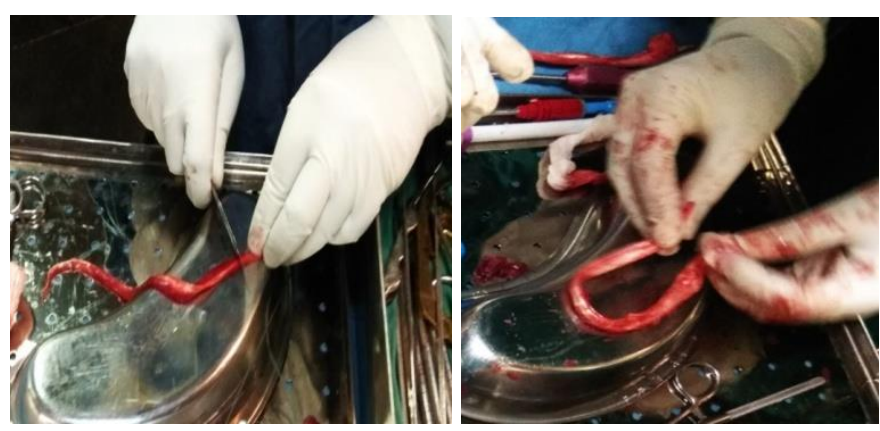

Image showing graft preparation

\section{Length measurement}

Graft length is measured, four stranded graft made by doubling two separate tendon.

Determining graft diameter: if tendon length is adequate, it may be cut half to make two separate fragment of equal length. Each segment is looped to form four stranded graft. It is passed from sizing device.
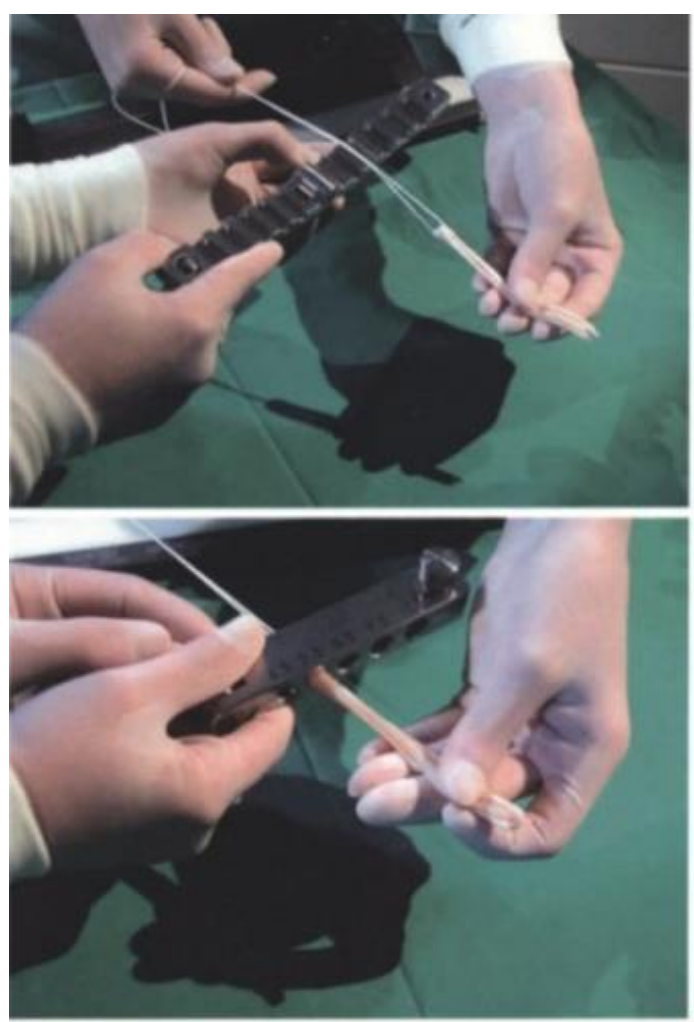

Image showing measuring of graft diameter using sizing device

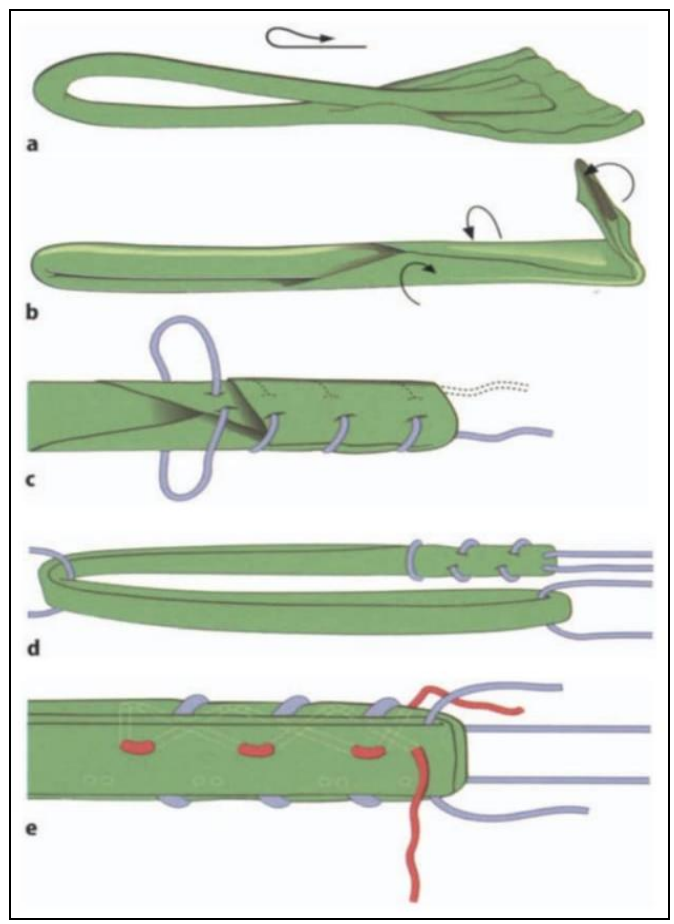

Folded tube technique ${ }^{[53]}$ : Jensen's quadrupled folded tube technique of tendon preparation, broader part is wrapped and tibial insertion end is secured with whip stich suture. This make graft with two loops. The loop is sutured to folded tube part of graft with two mersilene suture using criss cross technique.

\section{Debridement / notchoplasty}

After diagnostic arthroscopy the remnant part of PCL, soft tissue, fatpads are debrided using shaver. Notch of PCL foot pad is cleared. Local Synovitis, infrapatellar plica, fat pad anterior to PCL should be debrided with shaver. Large portion of PCL origin has debrided for satisfactory viewing of area.

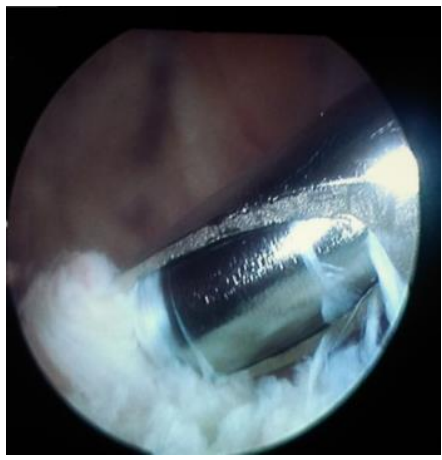

Image showing debridement / notch preparation using arthroscopic shaver

\section{Posterior Inspection}

Arthroscope is passed into posteromedial recess. Posteromedial portal is established. It is placed quiet above to see PCL origin adequately. Percutaneous needle is used to guide optimum site for placing posteromedial portal. PCL can be viewed by passing arthroscope to posteromedial portal. PCL origin site has debrided. Remnant PCL ligament is cleared.

Posterior joint debridement: Chronic synovitis, scar tissue may obstruct the tibial insertion of PCL. The capsule is dissected downward and posteriorly to expose PCL insertion and it provide more space for position of tibial drill guide. Posterior aspect is visualized and osteophytes are removed. 


\section{Femoral tunnel}

Femoral drill guide is used to mark the tunnel position. It is placed 11.30 to $12.30 \mathrm{O}$ ' clock position in left knee and 1.0 to2.O' clock position in right knee and 7 to $8 \mathrm{~mm}$ posterior to chondro-osseous junction. A low anterolateral instrument portal has been created and guide wire is passed from portal to marked point in tunnel and drilled into medial femoral condyle in 120 degree of knee flexion. Guide wire is over drilled to the graft size with reamer and using $4.5 \mathrm{~mm}$ cannulated drill. Length of femoral tunnel is measured using measuring device. Reaming s done very carefully to avoid breach the medial femoral cortex. The shaver is used to clear tibial tunnel inlet of bony debris.
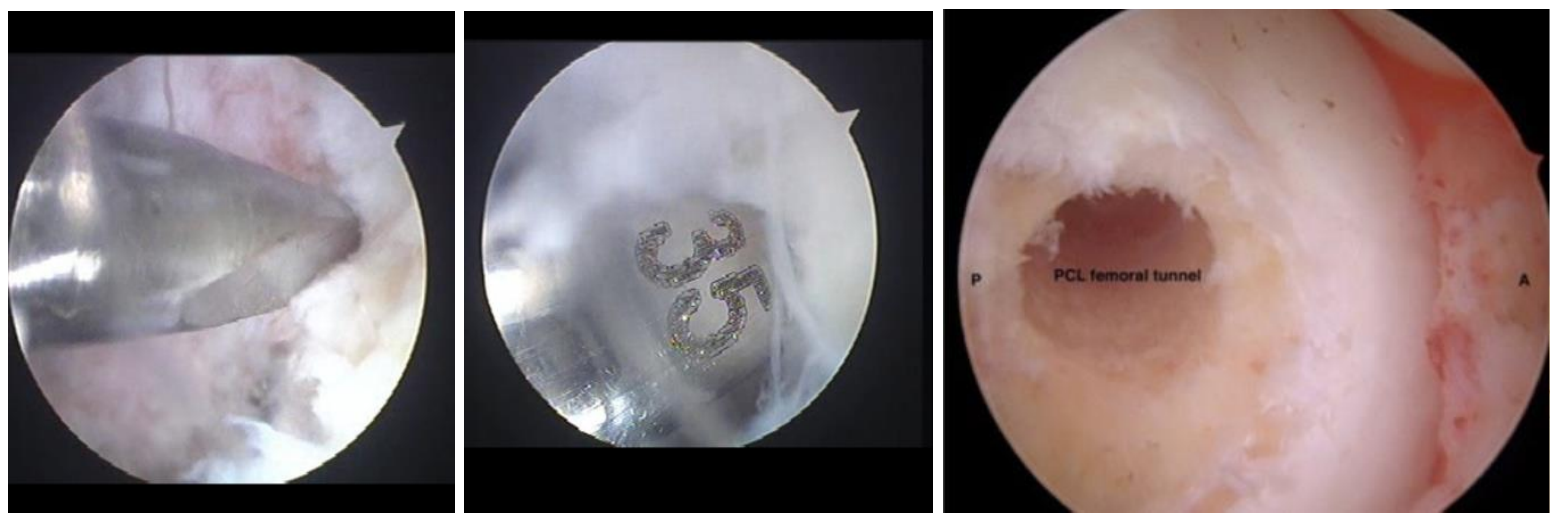

Image showing femoral tunnel preparation using inside out technique

\section{Tibial tunnel}

Tibialdrill guide (PCL ZIG) is advanced through anteromedial portal and placed on PCL insertion site of anterolateral bundle on posterior tibial surface. Position is monitored through posteromedial portal. Guide wire is placed and advanced. Origin is viewd by placing scope on posteromedial recess. Tibial drill guide passed from anteromedial and positioned at insertion site. The position is confirmed using image intensifier. Once the position of guide wire is satisfied. Reaming done using cannulated reamer. Length of tibial tunnel measured using reading in guide wire
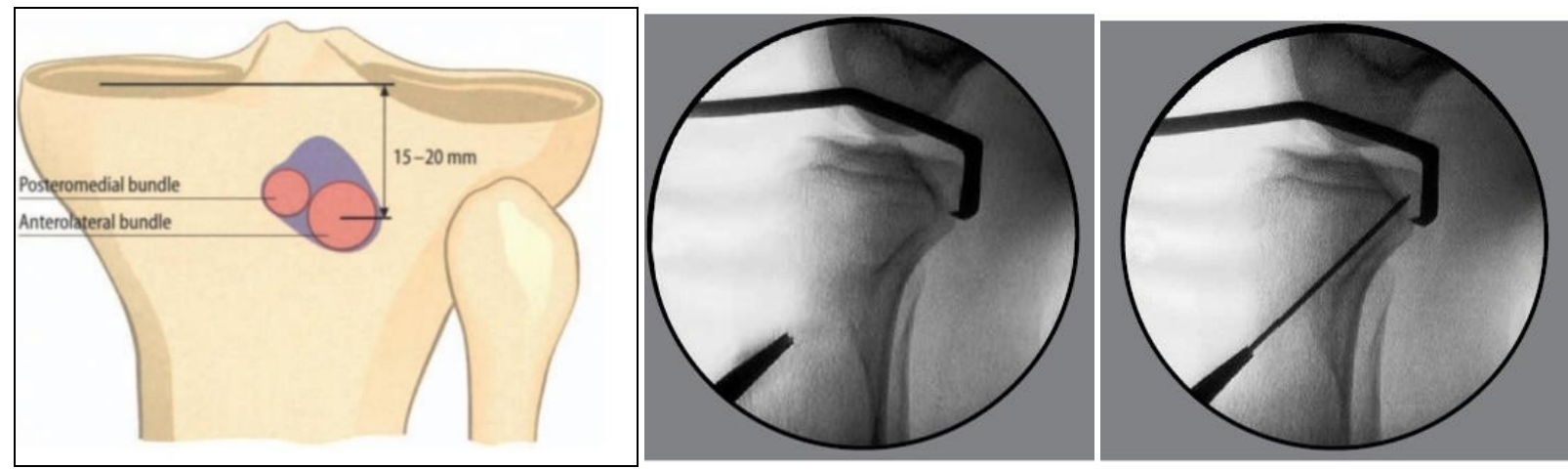

Image showing location of tibial tunnel placement, PCL tibial jig at posterior tibia and passage of guide wire

Tibial passing suture: Suture is passed from tibial tunnel and grasped from anteromedial portal using grasping forceps. The tibial passing suture is taken out through medial instrument portal.

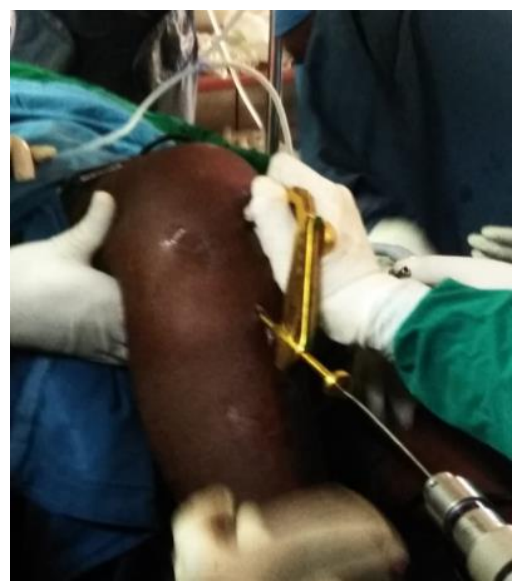

Image showing passing of guide wire from PCL tibial jig
Femoral passing suture: Suture is passed using guide wire which is advanced from anterolateral portal to create femoral tunnel. Suture is grasped from anteromedial portal and pulled out.

\section{Passage of graft, suture shuttling, fixation of graft}

Harvested graft is prepared, quadrupled, it loop end is attached to endobutton loop. The marking in the graft is done. The suture present in joint is pulled through tibial tunnel and it is attached with endobutton thread. Suture shuttling has been done. after shuttling, the graft is pulled into femoral tunnel. the graft is pulled till the marking is crossed, endobutton thread is pulled and flipped after satisfactory length of graft passed from joint. After flipping of endobutton the femoral graft is fixed with interference screw of measured diameter and length according to femoral tunnel diameter. The femoral graft fixation done in $90^{\circ} \mathrm{knee}$ flexion providing anterior drawer force to knee to overcome posterior translation. Tibial graft fixation is done in knee extension with interference screw of appropriate measured size and length. 

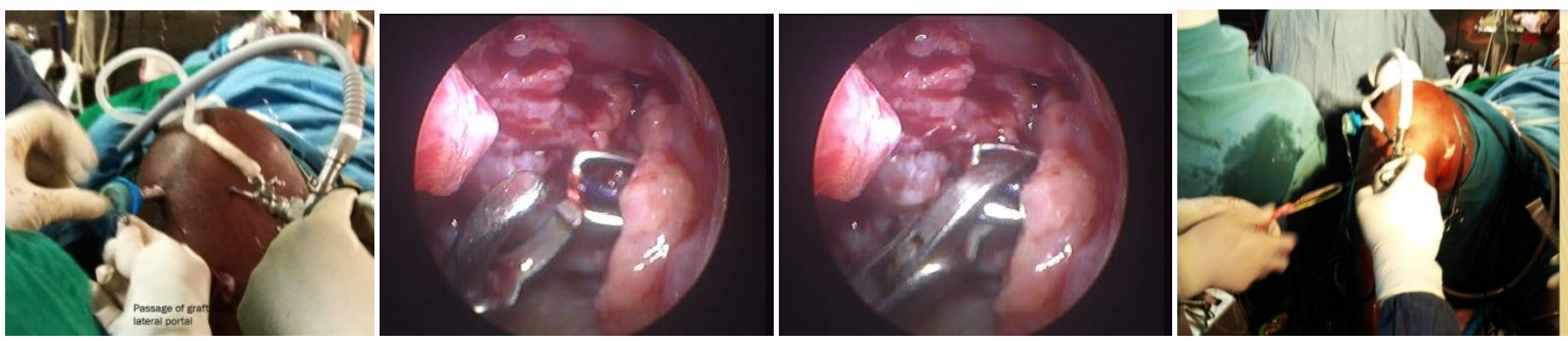

Clinical image showing passing of femoral suture, shuteling of suture and passing of graft

After fixation of graft arthroscopic visualization on graft has been done to look for impingement, laxity, and posterior drawer test is performed to check stability of graft. Other associated injury to knee planned for repair or reconstruction like posterolateral corner repair, ACL reconstruction is done accordingly.

The joint is irrigated, graft donor site is washed, portal site, graft harvesting site is closed in layer after drain is fixed in to joint. Sterile dressing is applied.

Distal pulse is checked. Patient is put on knee brace.

\section{Post-operative protocol}

The patient is shifted to ward. General condition, distal pulse, blood pressure is recorded. Post-operative IV antibiotic is given for 3 days. Patient is put on knee brace in full extension. Drain is checked for amount of drain, functioning of drain. The wound is inspected on $2^{\text {nd }}$ post-operative day and drain is removed, followed by $5^{\text {th }}$ and $7^{\text {th }}$ post-operative day dressing. Suture removal is done on $12^{\text {th }}$ to $14^{\text {th }}$ days depending on surgical wound status. Rehabilitation starts from post-operative day 1 .

Day 1: Analgesics and iv antibiotic, Drain care,Knee brace support, Ankle and toe movement exercise

Day 2: First sterile dressing, Removal of drain, Antibiotic and analgesics

Day 5: Second sterile dressing, IV antibiotics upto day 5

Day 8 Sterile dressing \& Wound care

Day 12: Suture removal

\section{Pcl Reconstruction Rehabilatation Protocol ${ }^{[54]}$}

\section{Week 0-2}

- weight bearing as tolerated with crutch or with locked brace in full extension

\section{Exercises}

- Leg raising exercise, Ankle pump, quadriceps exercise, Calf pumping, Passive extension to 0 degree

\section{2 -4 Week}

- Closed kinetic chain reaction, Active quadriceps work near full extension with resistance as tolerated, Weight bearing : gradually wean off crutches \& Range of motion : gradually increase range of motion within hinged brace up to 90 degree Limiting to 60 degree flexion during active and resisted work

\section{4-6 Weeks}

- Increase closed chain exercise -Isokinetic quadriceps exercise, $0-60^{0}$

- Static cycle with Weight bearing: full \&Range of motion : no limit to range of motion, Limiting to 60 degree flexion during active and resisted work

\section{6 - 12 weeks}

- Improve strength, power, indurence overall with Full range of movement

- Stepper, increasing intensity and duration as tolerated/ required \& Rower, gradually increase Resistance as tolerated

\section{Month 3- 6}

- open kinetic chain hamstring work, adding resistance / speed of contraction/ repetitions as progression, Low speed isokinetics, Swimming, Dynamic hydrotherapy, including pool running Early plyometrics \&Light agility

\section{Month 6-9}

- Non predicatable agility training, Introduce high speed Isokinetics, Running, Plyometric training, Sport specific training

\section{Month 9}

Goals

- Unrestricted confident function \& Earliest return to sports

\section{Evaluation}

All the patient treated in this study were followed up at 6 weeks, 3 month, 6 month and 9 month and 1 year. Postoperatively knee $\mathrm{x}$ ray, anteroposterior and Lateral view taken to look the location of interference screw and endobutton. Patients were explained detail about rehabilitation protocol, exercises to be done, weight bearing detail, use of crutch, exercises to be done at home. Patients are instructed to come for follow up in instructed duration to look for improvement, any compalication, range of motion, and rehabilitation details.

The evaluation of functional outcome is done using IKDC score, LYSHOLM score, TAGNER score and PCL stress xrays.

IKDC score ${ }^{[54]}$ : Is International Knee Documentation Score. it is a subjective scale score which gives patients overall function score. It looks at three category namely Symptoms, sports activity \& knee function.

Lysholm knee scoring scale ${ }^{[55]}$ : It is form of questionnaire responded by patient. Each possible response has been given an arbitrary score on an increasing scale. Maximum score is sum of score in response given by patient.

Lysholm questionnaire contain eight parameters including Limp, Support, Locking, Instability, Pain, Swelling, Stair climbing \& Squatting 
Tegner activity level scale ${ }^{[56]}$ : It is a scale which give standardized method of grading work and sports activity. It is one item score which grade the activity based on work and sports activity. Scale from zero to 10 . Zero level represents disability and 10 shows international or national level sports person or soccer.

PCL stress view $x$ ray: PCL stress view $x$ ray was taken pre operatively and on follow up visits. The posterior translation was measured and compared pre operatively and on post operative follow up visits.

\section{Results}

20 patients were operated with posterior cruciate ligament reconstruction They were followed up and evaluated using Ikdc Score, Lysholm Knee Score And Tegner Activity Knee Score and PCL stress xray measuring posterior translation( PT) of tibia in operated patients during follow up in Government Stanley Medical college and Hospital Chennai between June 2017 to
October 2019. AGE Distribution

AGE Distribution

\begin{tabular}{|c|c|c|}
\hline Age Group & Male & Female \\
\hline $20-25$ year & 5 & $25 \%$ \\
\hline $26-30$ year & 6 & $30 \%$ \\
\hline $31-35$ year & 2 & $10 \%$ \\
\hline $36-40$ year & 3 & $15 \%$ \\
\hline $41-45$ year & 3 & $15 \%$ \\
\hline $46-50$ year & - & - \\
\hline $51-55$ year & 1 & $5 \%$ \\
\hline
\end{tabular}

Most of patients belong to age group 26 - 30 year and followed by age group $20-25$ year.

\section{Gender Distribution}

Male patients $(95 \%)$ are predominant in having knee injury than female patients $(5 \%)$.

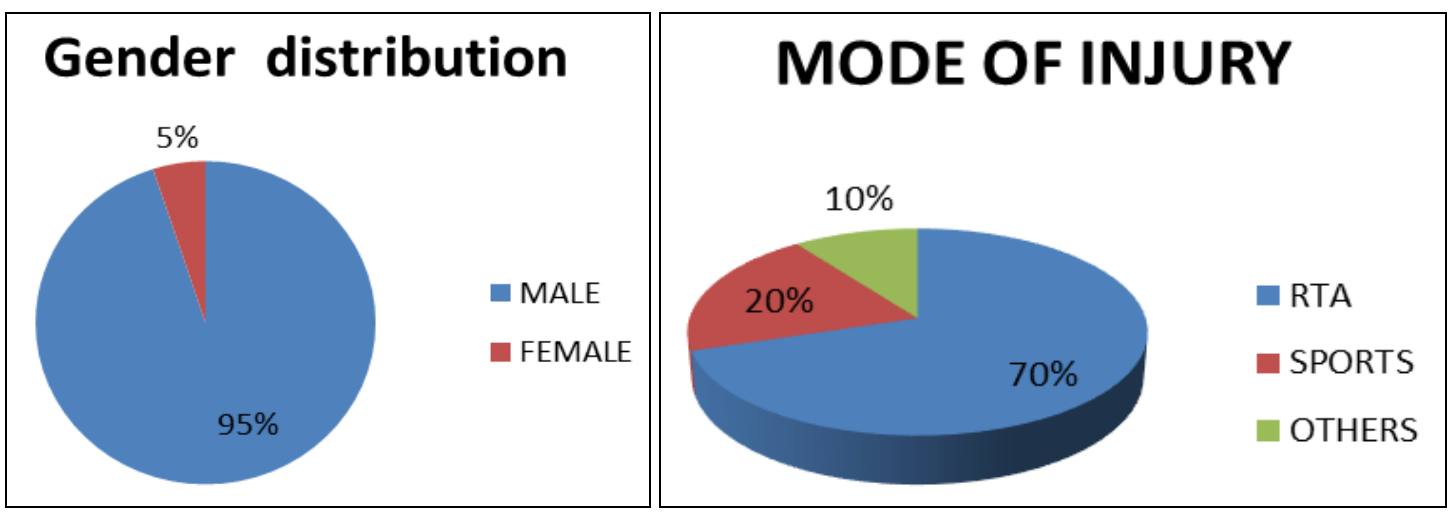

\section{Mode of Injury}

Road traffic accident is most common mode of injury to the patients (70\%) followed by sports injury.

Injury Duration

\begin{tabular}{|c|c|c|}
\hline Duration & Patients & Percentage \\
\hline Up to 2 months & 5 & $25 \%$ \\
\hline $3-4$ months & 10 & $40 \%$ \\
\hline $5-6$ months & 4 & $20 \%$ \\
\hline $7-8$ months & 1 & $5 \%$ \\
\hline $8-9$ months & - & - \\
\hline$>9$ months & - & - \\
\hline
\end{tabular}

Most patients belong to injury duration between $3-4$ months (40\%).

\section{Associated Injuries}
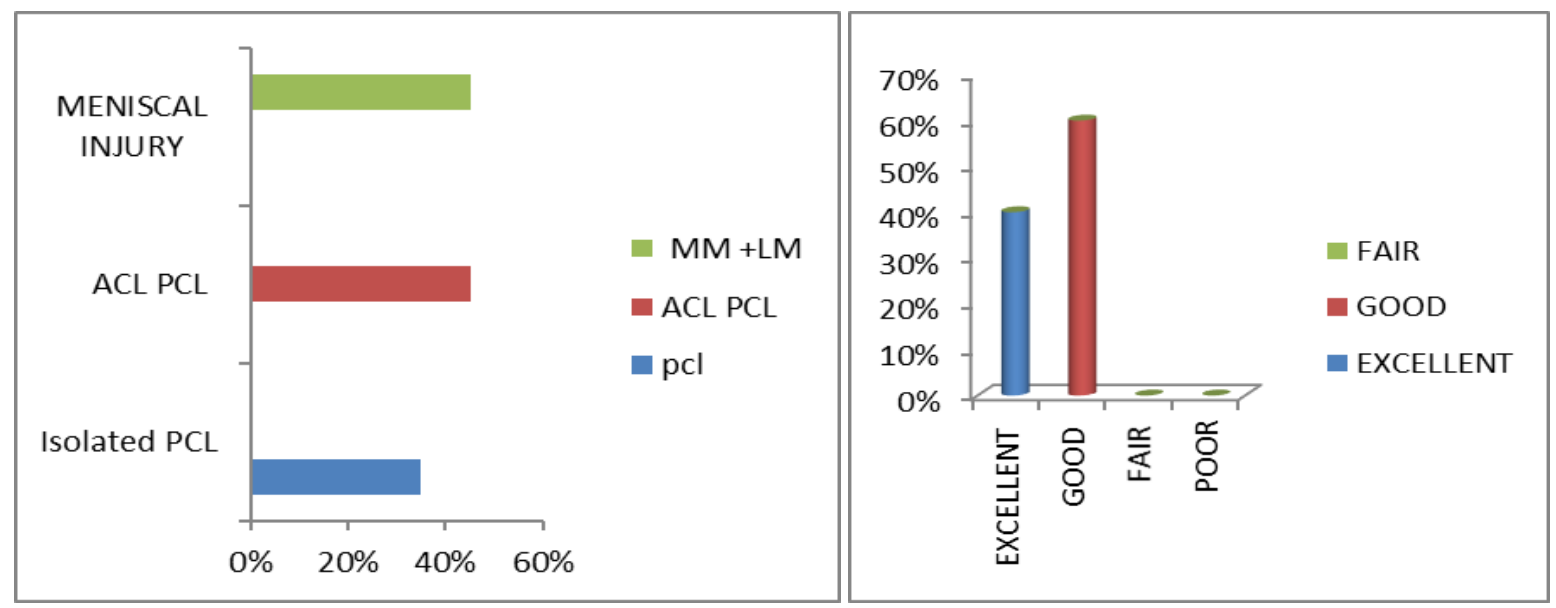
$35 \%$ patientssustained isolated PCL injury. $45 \%$ patients sustained multi-ligament injury. $45 \%$ of patients sustained meniscus injury.

\section{Lysholm Knee Score}

$40 \%$ had excellent \& $60 \%$ had good outcome

Ikdc Subjective Score

\begin{tabular}{|l|c|c|}
\hline & Pre-operative score & Post-operative score \\
\hline IKDC subjective score & 46.75 & 92.3 \\
\hline
\end{tabular}

Tegner Activity Score

\begin{tabular}{|c|c|c|}
\hline & Mean Pre op score & Mean Post op score \\
\hline Tegner activity score & 3.95 & 7.25 \\
\hline
\end{tabular}

There is significant improvement in tegner activity score from mean 3.95 to post 7.25 in follow up post operatively.

PCL Stress View X Ray

\begin{tabular}{|c|c|c|}
\hline & $\begin{array}{c}\text { Mean pre op posterior } \\
\text { translation }(\mathbf{m m})\end{array}$ & $\begin{array}{c}\text { Mean post op posterior } \\
\text { translation }\end{array}$ \\
\hline PT & $11.58 \mathrm{~mm}$ & $3.72 \mathrm{~mm}$ \\
\hline
\end{tabular}

\section{Complication}

One patient developed superficial infection at graft donor site which is treated with dressing and antibiotic.

One patient had knee stiffness due to poor follow up of patients. Patient is given physiotherapy, patient improved with physiotherapy exercises.

\section{Discussion}

In 1845, Amedee described three signs which are suggestive of ligament injury. These were snapping noise, hemarthrosis and loss of function of the knee.

In 1903, A.W. Mayo Robson ${ }^{[4]}$ of lead England published the first known report of repair of anterior and posterior cruciate ligament in association with repair of other ligaments in an acutely dislocated knee.

The first reports on reconstruction of posterior cruciate ligament was published in 1917 and 1919 by Hey Groves ${ }^{[5]}$. He used semitendinosus graft. Hesutured the proximal end of semitendinosus to the semimembranosus posteriorly at level of tibia. Gallie and Lemesurier ${ }^{[6]}$ reported a modification of Hey Groves reconstruction in 1926 and demonstrated successful reconstruction of PCL in their case report.

In 1936, Bosworth described an extra articular method of reconstruction of cruciate by using fascia lata grafts externally crisscrossed from the femoral tibial condyle ${ }^{[7]}$. In1937 Cubbins et al. ${ }^{[8]}$ reported approximately five cases of posterior cruciate ligament reconstruction in which they used posterior strip of iliotibial band. The tibial attachment of iliotibial band is preserved and harvested ITB is looped around the fibular head deep to lateral gastrocnemius tendon and through the posterior capsule it was attached to medial femoral condyle by drilling a hole. They reported considerable improvement in these knees with this procedure ${ }^{[9]}$.

In 1938, Palmer, in his thesis report of three cases of posterior cruciate ligament in which he used central or inner half of semimembranous tendon preserving the tibial attachment graft passed and fixed it into intercondylar notch and through a drill hole on medial femoral condyle ${ }^{[10]}$. Others subsequently have given case report of use of this same type of reconstruction.

In 1950 Lindmann $^{11}$ reported a method of reconstruction of PCL in which he detached the semitendinosus from its tibial insertion and passed it through medial femoral condyle through notch and posteriorly through the tibia to anteromedial face of tibia. In 1968, Du Toit reported on his use of the Lindman procedure in two cases, He stated that reconstruction were found to be firm several years later. In 1968 Trickey ${ }^{[12]}$ reported on repair of 10 cases of posterior cruciate ligament instability wherein the only lesion was acute avulsion fracture of posterior cruciate ligament at tibial insertion. All 10 cases he reported were successful ${ }^{[7]}$.

Torisu [13] in 1977 published a report advocating Trickey's method of open reduction of bone fragment followed by internal fixation especially in cases where the fragment was displaced. Hoghston called the strongest ligament of knee, PCL as 'central stabilizer' and 'key to the knee joint". In1981, Loose et al. found only 102 cases of PCL injury in more than 13000 knee operation. Neurath (1992) ${ }^{[14]}$ : PCL has considerably richer blood supply than the ACL and thus correlates with its high content of type IV collagen. Odensten (1993) and Neurath (1992) told that femoral origin of PCL forming an elliptical area that extend about $20 \mathrm{~mm}$ posteriorly. This area has a transverse diameter of approximately $10 \mathrm{~mm}^{[15]}$.

In 1993, Back surveyed 55 experienced specialists in sports medicine who treated professional and Olympic athletes or they were the directors of sports clinic. This survey result document the fact that patient with PCL injuries and surgeons who treats them constitute a "Fringe group" in field of orthopedics trauma surgery ${ }^{[16]}$

In 1994, Race ${ }^{[17]}$ stated that anterolateral bundle is primarily responsible for overall stabilizing effect of PCL. In 1994,Covey ${ }^{[18]}$ stated that intrasynovial vessels forms a branching network of perligamentous vessels which give off small connecting branches that penetrates the PCL and anastomoses with network of intraligamentous vessels.

Fanelli (1994) showed that PCL tear occurs upto38.3\% of acute knee injuries. Harner (1995) cited a frequency of $3.5 \%$ to $10 \%$ of knee injuries has PCL tear. Harner (1995) and Van dommelen (1989) suggested that meniscofemoral ligament have a better elastic modules than posteromedial fibre system of PCL ${ }^{[19]}$.

Wong $\mathrm{T}$ et al. ${ }^{[20]}$ concluded that $\mathrm{AM}$ and $\mathrm{AL}$ trans tibial arthroscopic PCL reconstruction produced comparable clinical result in short term follow up. Sekiya JK et $a l^{21}$ concluded that single bundle PCL reconstruction produces a satisfactory return of function and improvement in symptoms.

Chan YS et al. ${ }^{[22]}$ concluded that in long term follow up of more than 3 years, the analytical result shows satisfactory function after PCL reconstruction. They suggested that hamstring graft tendon is effective, safe and acceptable.

Chatila $\mathrm{J}$ et al. ${ }^{[23]}$ concluded that knee stability and satisfactory outcome were achieved with both single bundle and double bundle PCL reconstruction surgery. Kim et al. ${ }^{[24]}$ in his study on clinical results after single bundle PCL reconstruction concluded that there is significant improvement in posterior knee laxity and Lysholm score and up to $75 \%$ patients had near normal subjective function on IKDC score.

Bjarne Mygind-Klavsen [25], MD, Torsten Gronbech Nielson, PT, and Martin Lind, in their study concluded that there is no difference in final outcomes found in final follow up for patients having isolated PCL injury and multi ligament knee injury.

Fanelli GC, Edsom CJ ${ }^{[26]}$ In their case series study concluded that combined ACL and PCL injury can be treated with arthroscopic reconstruction and appropriate collateral ligament surgery in long term follow up the knees are functionally stable. Jung TM ${ }^{[27]}$, Lubowicki A, Wienand A, Wagner M, Weiler A, In their study concluded that female patients will have superior 
reduction of knee laxity after posterior cruciate ligament reconstruction compared to male patients.

Kim YM, Lee CA, Matava MJ [28], On their study on result of arthroscopic single bundle transtibial posterior cruciate ligament reconstruction, concluded that isolated PCL tear can improve posterior knee laxity by grade I. $75 \%$ patients were having normal objective score.

PCL injury is less in incidence than ACL injury. Due to increase interest and involvement in sports, athletics activity, increased trend in using motor vehicle increase the risk of knee injuries are increased due to it. Arthroscopic reconstruction of PCL ligament using hamstring graft is standard treatment for patients having knee laxity, joint instability. Learning curve of PCL reconstruction is steep and it is more skill demanding.

In our study we used to reconstruct the single bundle of posterior curuciate ligament that is posterolateral bundle using hamstring auto graft. Single bundle and double bundle reconstruction are having similar functional outcome in long term follow up. Although double bundle reconstruction is biomechanically superior but it is quite complex procedure compared to single bundle reconstruction.

Hamstring graft is used for reconstruction as it is easy and simple to harvest and it has less donor site morbidity and less post operative pain associated with it. Patients rehabilitation is easy with hamstring graft. Quadrupled hamstring graft will have tensile strength near $4000 \mathrm{~N}^{\text {[57] }}$. Othergraft option available are bone patellar tendon graft, quadreiceps tendon, peronius longus, artificial grafts are available nowadays.

Our study is to evaluate the functional outcome of posterior cruciate ligament reconstruction.

This is a prospective study done in Government Stanley medical college Chennai to evaluate the functional outcome of reconstruction of PCL.

In our study 20 patients were operated. Among them male patients predominate with $95 \%$ and $5 \%$ were female patients. Right side knee injury (75\%) was more common than left side knee injury (25\%). Road traffic accident is the most common mode of injury causing PCL tear followed by sports injury and accidental fall. Most patients belongs to age group 26- 30 years, followed by $20-25$ years age group.

Average duration of follow up was 15.25 months with maximum follow up of 25 months and minimum follow up of 7.5 months.

There was significant improvement in average Lysholm score up to 92.15 post operatively from 48.1 preoperatively.

\begin{tabular}{|c|c|}
\hline Study & Average Lysholm score \\
\hline Fenelli G et al. ${ }^{[58]}$. & 91.7 \\
\hline Rocheconger G et al. ${ }^{[59]}$ & 90 \\
\hline Mariani PP, et al. ${ }^{[60]}$ & 95.1 \\
\hline Sung jae kim et al. ${ }^{[61]}$ & 89.2 \\
\hline Our study & 92.15 \\
\hline
\end{tabular}

There was significant improvement in IKDC score preoperatively from 46.75 to 92.3 post operative follow up.

\begin{tabular}{|c|c|}
\hline Study & IKDC score \\
\hline Fenelli GC et al. & 88.7 \\
\hline Chen P, et al. ${ }^{[2]}$ & 89.33 \\
\hline Rocheconger & 89 \\
\hline Our study & 92.3 \\
\hline
\end{tabular}

There was significant improvement in Tegner activity score from 3.95 to 7.25 postoperatively.

\begin{tabular}{|c|c|}
\hline Study & Tegner score \\
\hline Wu CH et al. $^{[63]}$ & 6 \\
\hline Chan YS et al. $^{[64]}$ & 6.3 \\
\hline Mariani PP et al & 6.9 \\
\hline Our study & 7.25 \\
\hline
\end{tabular}

There was significant improvement in knee laxity which was $11.58 \mathrm{~mm}$ average preoperatively and it improves and reduced up to $3.72 \mathrm{~mm}$ on follow up.

In our study one patient developed superficial infection in graft donor site which was treated with dressing and one patient developed knee stiffness due to irregular follow up and poor compliance which was improved by physiotherapy and knee mobilization exercise.

\section{Case 1}

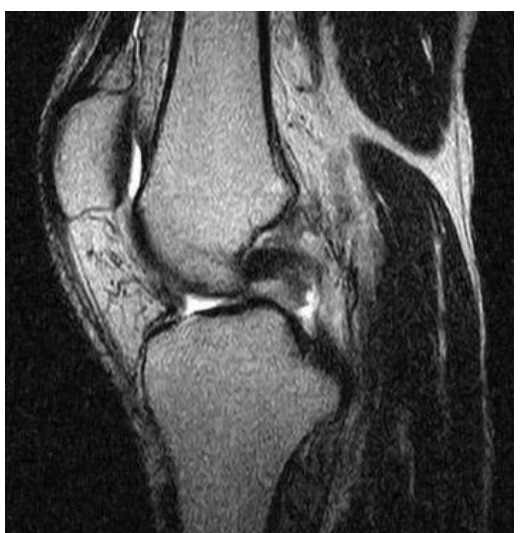

Fig 1: MRI image showing isolated PCL tear
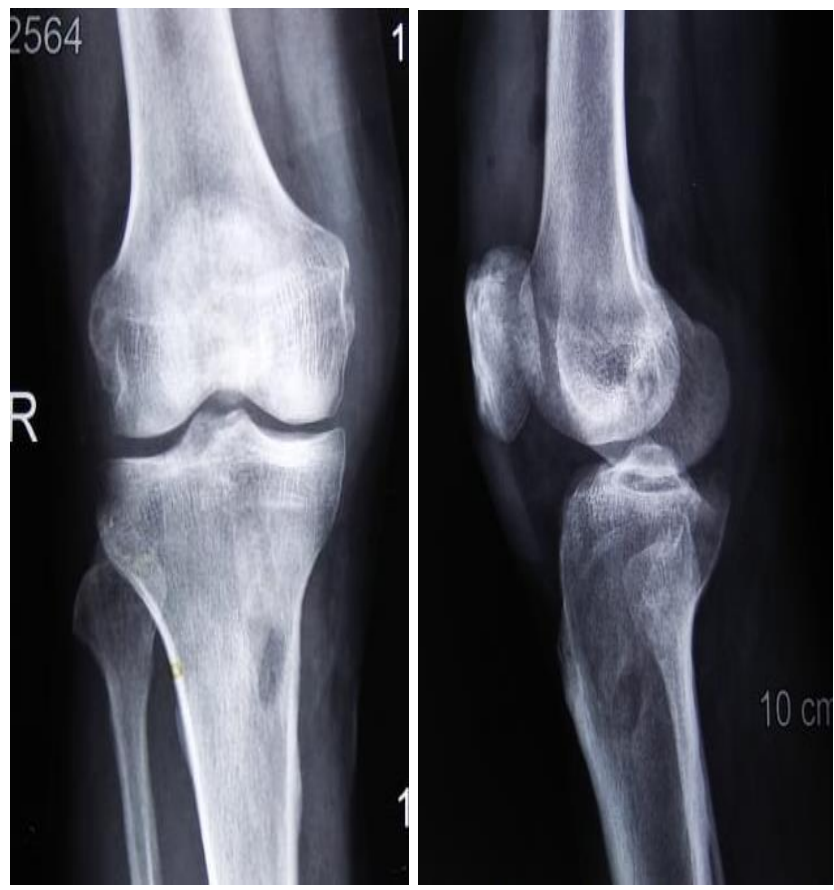

Fig 2: Showing post operative $\mathrm{x}$ ray after PCL reconstruction using biodegradable interference screw. 

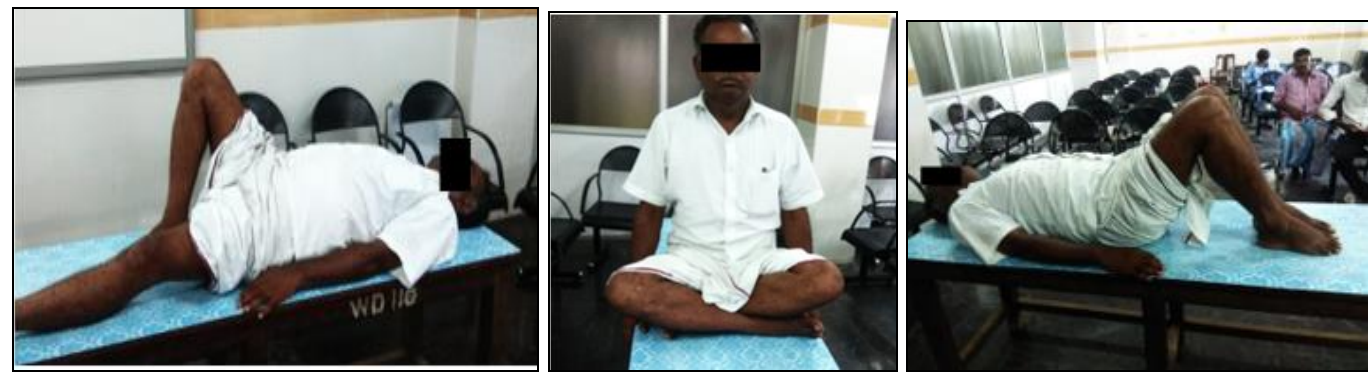

Fig 3: Patients showing good range of knee flexion able to cross leg sit and minimum knee laxity,

\section{Conclusion}

- In young active individuals, arthroscopic reconstruction of PCL injury has excellent and good functional outcome. Using quadripuled semi tendinoses as graft makes less post operative pain and less donor site morbidity and weakness. Rehabilitation is easier with hamstring graft.There is significant improvement in function and symptoms of knee post operatively with arthroscopy reconstruction.

- There is significant improvement in knee laxity post operatively.

\section{Limitation of study}

- Small sample size as PCL injury is less common injury.

- Subjective score was used to assess the result of study and not the objective score.

- Small follow up duration will limit the long term outcome and result of this procedure.

\section{Reference}

1. Gelber AC et al. joint injury in young adult and risk for subsequent knee and hip osteroarthristis. American journal of epidemiology 1989;10(2):278-88

2. Sheng Hao WANG et al. PLoS one 2018;13(10):e0205118 ,4,5,6,7,8,9,10,11,12,13,14: Jack.C. Hughston, MD, Phd, History of posterior cruciate ligament injury. Operative technique in sports medicine 1993;1(2):86-88

3. Micheal J Strobel, posterior crucitae ligament 2(15):572

4. Bach Micheal J Strobel, posterior crucitae ligament 1993;2(15):573

5. Race. Micheal J Strobel, posterior cruciate ligament 1994;2(15):573

6. Covey. Micheal J Strobel, posterior crucitae ligament 1994;2(15):572.

7. Harner, Von Dommelen. Micheal J Strobel, Posterior Crucitae Ligament 2(15):572

8. Wong $\mathrm{T}$ et al, arch orthop trauma surgery 2009

9. Sekiya JK et al, arthroscopy 2005

10. Chan YS et al, arthroscopy 2006

11. Chatila J et al, arthroscopy 2017.

12. Kim YM, LeeCA, Matava MJ. Clinical result of arthroscopic single bundle transtibial posterior cruciate ligament reconstruction: a systemic review. Am J Sports mrd 2011;39:425- 434.

13. Bjarne Mygind-Klavsen. Orthop J sports med 2017 apr 5(4):2325967117700077

14. Fanelli GC, Edson CJ. Arthroscopic assisted combined anterior and posterior cruciate ligament reconstruction in multiple ligament injured knee : 2-10 year follow up. arthroscopy 2002;18(7):703-14.

15. Jung TM et al. arthroscopy 2008

16. Kim YM, Lee CA, Matava MJ, AM J sports med. 2011;39(2):425-34.
17. Micheal J Strobel, Posterior Crucitae Ligament 2.15572

18. Histology Micheal J Strobel Anterior Cruciate Ligament Chapter Chapter 2.13

19. Micheal J Strobel Anterior Cruciate Ligament Chapter 2.13

20. Micheal J Strobel, General Part Historical Development Chapter 1, 4

21. Micheal J Strobel, General Part Historical Development Chapter 1:4 -5

22. Kennedy Micheal J Strobel

23. Micheal J Strobel cooper classification,posterior cruciate ligament chapter 2(15):573

24. Natural history campbells operative orthopaedics knee injury

25. ligament healing: campbells operative orthopedics, sports medicine part xiii: 2157

26. Non operative treatment PCL knee injury chapter 45 page 2239 campbell's operative orthopaedics

27. Treatment algorithm of PCL injury Knee Injury chapter campbell's operative orthopaedics 45, 2241

28. Mcginty, Johnson. general principle of arthroscopy : campbells operative orthopedics chapter 49:2458

29. Micheal J Strobel anterior cruciate ligament chapter 2(13): 398-400

30. Micheal J Strobel special part chapter 2, 384-389.

31. portal in arthroscopy campbells operative orthopedics, sports medicine xiii, 2488-2489

32. Diagnostic arthrosopy of knee, campbells operative orthopedics, sports medicine xiv, 2490

33. graft harvesting Michael j. strobe posterior cruciate ligament chapter 2(15):599

34. Folded tube technique: Micheal J Strobel anterior cruciate ligament chapter 2(13):433.

35. rehabilitation protocol, post-operative rehabilitation following PCL reconstruction surgery. The sports and knee clinic, Wrightingon hospital

36. IKDC score Annexure

37. Lysholm score Annexure

38. Tegner score Annexure

39. Manual of Arthroscopic Surgery Michael j strobel, posterior cruciate ligament chapter 2(15)

40. Fenelli G et al. Arthroscopy 2002;18:703-714 Sports Medicine and Arthroscopy Review: December 2018;26(4):150-152 doi: 10.1097/JSA.0000000000000220

41. Rocheconger $\mathrm{G}$ arthroscopy 2011.

42. Ferretti A, Papandrea P, Conteduca F, Mariani PP. The American journal of sports medicine 20(2):203-207

43. Anterior Cruciate Ligament Reconstruction in Patients with Generalized Joint

44. Laxity, Sung-Jae Kim, MD, Praveen Kumar, MS, ${ }^{*}$ and Sung-Hwan Kim, MD Clin Orthop Surg 2010;2(3):130

45. Chen $\mathrm{P}$ et al. orthopedics 2012;27(6):851-6. doi: 
10.1016/j.arth.2012.01.011. Epub 2012 Mar 3.

46. Arthroscopic reconstruction of the posterior cruciate ligament by using a quadriceps tendon autograft: a minimum 5-year follow-up

47. Chin-Hsien Wu' Alvin Chao-Yu Chen, Li-Jen Yuan, ChungHsun Chang, Yi-Sheng Chan, Kuo-Yao Hsu, et al, Journal of Arthroscopy 2007;23(4):420-7. doi: 10.1016/j.arthro.2006.12.011

48. Arthroscopic reconstruction of the posterior cruciate ligament with use of a quadruple hamstring tendon graft with 3- to 5-year follow-up

49. Yi-Sheng Chan, Shih-ChiehYang, Chung-Hsun Chang, Alvin Chao-Yu Chen, Li-Jen Yuan, Kuo-Yao Hsu, et al, Journal of Arthroscopy 2006;22(7):762-70. doi: 10.1016/j.arthro.2006.03.020 